\title{
Neuroprotective Effects of Resveratrol in Ischemic Brain Injury
}

\author{
Noelia D. Machado 1,2,*, Gorka Villena Armas ${ }^{3}$, Mariana A. Fernández ${ }^{1,4} \mathbb{D}$, Santiago Grijalvo ${ }^{5,6} \mathbb{D}$ \\ and David Díaz Díaz $3,7,8, *$ (D)
}

1 Facultad de Ciencias Químicas, Departamento de Química Orgánica, Universidad Nacional de Córdoba, Ciudad Universitaria, Córdoba X5000HUA, Argentina; marfer@fcq.unc.edu.ar

2 Instituto de Investigación y Desarrollo en Ingeniería de Procesos y Química Aplicada (IPQA-UNC-CONICET), Av. Vélez Sarsfield 1611, Córdoba X5016GCA, Argentina

3 Instituto Universitario de Bio-Orgánica Antonio González, Universidad de La Laguna, Avda, Astrofísico Francisco Sánchez 2, 38206 La Laguna, Tenerife, Spain; alu0101017471@ull.edu.es

4 Instituto de Investigaciones en Físico-Química de Córdoba (INFIQC-CONICET), Ciudad Universitaria, Córdoba X5000HUA, Argentina

5 Institute for Advanced Chemistry of Catalonia (IQAC-CSIC), Jordi Girona 18-26, 08034 Barcelona, Catalonia, Spain; sgrgma@cid.csic.es

6 Biomedical Research Networking Center in Bioengineering, Biomaterials and Nanomedicine, CIBER-BBN, Jordi Girona 18-26, 08034 Barcelona, Catalonia, Spain

7 Departamento de Química Orgánica, Universidad de La Laguna, Avda. Astrofísico Francisco Sánchez S/N, 38206 La Laguna, Tenerife, Spain

8 Institut für Organische Chemie, Universität Regensburg, Universitätsstr. 31, 93053 Regensburg, Germany

* Correspondence: nmachado@fcq.unc.edu.ar (N.D.M.); ddiazdiaz@ull.edu.es (D.D.D.); Tel.: +54-0351-5353687 (N.D.M.); +34-922318610 (D.D.D.)

Citation: Machado, N.D.; Armas, G.V.; Fernández, M.A.; Grijalvo, S.; Díaz Díaz, D. Neuroprotective Effects of Resveratrol in Ischemic Brain Injury. NeuroSci 2021, 2, 305-319. https: / / doi.org/10.3390/ neurosci2030022

Academic Editor: Masato Nakafuku

Received: 1 August 2021

Accepted: 26 August 2021

Published: 1 September 2021

Publisher's Note: MDPI stays neutral with regard to jurisdictional claims in published maps and institutional affiliations.

\section{Copyright: (c) 2021 by the authors.} Licensee MDPI, Basel, Switzerland. This article is an open access article distributed under the terms and conditions of the Creative Commons Attribution (CC BY) license (https:/ / creativecommons.org/licenses/by/ $4.0 /)$.
Abstract: Cerebral ischemia represents the third cause of death and the first cause of disability in adults. This process results from decreasing cerebral blood flow levels as a result of the occlusion of a major cerebral artery. This restriction in blood supply generates low levels of oxygen and glucose, which leads to a decrease in the energy metabolism of the cell, producing inflammation, and finally, neurological deterioration. Currently, blood restoration of flow is the only effective approach as a therapy in terms of ischemic stroke. However, a significant number of patients still have a poor prognosis, probably owing to the increase in the generation of reactive oxygen species (ROS) during the reperfusion of damaged tissue. Oxidative stress and inflammation can be avoided by modulating mitochondrial function and have been identified as potential targets for the treatment of cerebral ischemia. In recent years, the beneficial actions of flavonoids and polyphenols against cerebrovascular diseases have been extensively investigated. The use of resveratrol (RSV) has been shown to markedly decrease brain damage caused by ischemia in numerous studies. According to in vitro and in vivo experiments, there is growing evidence that RSV is involved in several pathways, including cAMP/AMPK/SIRT1 regulation, JAK/ERK/STAT signaling pathway modulation, TLR4 signal transduction regulation, gut/brain axis modulation, GLUT3 up-regulation inhibition, neuronal autophagy activation, and de novo SUR1 expression inhibition. In this review, we summarize the recent outcomes based on the neuroprotective effect of RSV itself and RSV-loaded nanoparticles in vitro and in vivo models focusing on such mechanisms of action as well as describing the potential therapeutic strategies in which RSV plays an active role in cases of ischemic brain injury.

Keywords: blood-brain barrier; brain; ischemia; neuroprotection; oxidative stress; polyphenols; reperfusion; resveratrol; therapy

\section{Introduction}

Resveratrol (RSV) is a natural stilbene class of polyphenol (trans-3,5, $4^{\prime}$-trihydroxystilbene) present in a large variety of vegetal species, such as grapes, mulberries, peanuts, and pomegranates [1]. In recent years, the scientific community has been very interested in this compound due to its broad range of potential biological activities. In particular, important 
therapeutic effects have been related to this polyphenol administration as antioxidant [2], anti-inflammatory [3], cardioprotective [4], and anti-carcinogenic [5], among others [6,7].

RSV has been studied for the treatment of multiple diseases such as obesity, diabetes, cardiovascular problems [8]. Additionally, evaluation of its effects as a therapeutic agent on neuroprotection and nerve regeneration has gained special relevance in recent years [9]. For instance, RSV could mediate nerve regeneration and motor repair of sciatic nerve crush injury in a rat model [10] as well as improve spinal cord injury [11].

Stroke is a cerebrovascular disease caused by the interruption of blood flow to the brain due to the blockage or rupture of a vessel. Approximately $80 \%$ of all strokes are ischemic, and there are limited therapies approved for the treatment of acute ischemic stroke. Several natural compounds have been used for the prevention of strokes [12] and others that produce a protective action on the cardiovascular system, including RSV [13,14]. For example, atherosclerosis is considered a risk factor for ischemic stroke. In this sense, RSV could inhibit the platelet activation and aggregation induced by collagen, adenosine diphosphate, and thrombin. The proposed mechanism involved the inhibition either of tissue factor gene expression or the synthesis of prothrombotic mediators [15]. Cerebral edema and intracranial hypertension are common complications of cerebral infarction and the major causes of mortality. A range of therapeutic agents that successfully target cerebral edema have been developed in animal studies, some of which have been assessed in clinical trials; between them, RSV has been proved as a therapeutic agent $[16,17]$. Although RSV is a highly hydrophobic molecule, the penetration to a membrane such as the blood-brain barrier (BBB) is extremely difficult. However, an alternative administration is via the nasal in the olfactory region, resulting in a more comfortable route for the patient [18]. RSV has several mechanisms of action linked to their effects against stroke, as the molecule interacts with a wide range of enzymes and receptors, improving the resistance to stress and reducing apoptosis [19].

Brain damage and neuronal death after an acute central nervous system (CNS) injury such as stroke are synergistically mediated by many pathophysiologic mechanisms that include oxidative stress, inflammation, and ionic imbalance, besides apoptosis. As was mentioned, the treatment with RSV has been shown to prevent or slow down many of these pathological changes [20]. Polyphenols also exert neuroprotective actions when administered after stroke, indicating that these molecules may be helpful in the recovery of stroke patients [21] and neonatal hypoxic-ischemic brain injuries [22,23]. There are several recent reviews dealing with the neuroprotective mechanisms produced by natural polyphenolic compounds $[18,24,25]$. Antioxidants such as RSV generally work as ROS scavengers and metal chelators due to the presence of hydroxyl groups [26]. It has been proposed that beneficial actions of the activated Sonic hedgehog (Shh) signaling pathway, such as improved neurological function, decreased infarct volume, enhanced vitality, and reduced apoptosis of neurons after stroke, can be produced by RSV [27]. Wan et al. reported that RSV also provides neuroprotection by inhibiting phosphodiesterase (PDEs) and regulating the cyclic adenosine monophosphate (cAMP)/adenosine monophosphate (AMPK)/sirtuin 1 (SIRT1) pathway, which reduces ATP energy consumption during ischemia [28].

This review summarizes the effects of RSV in nerve regeneration after an ischemic brain injury episode (Figure 1), and it is focused on the published results since 2015, complementing various previous revisions $[19,20,29]$. 


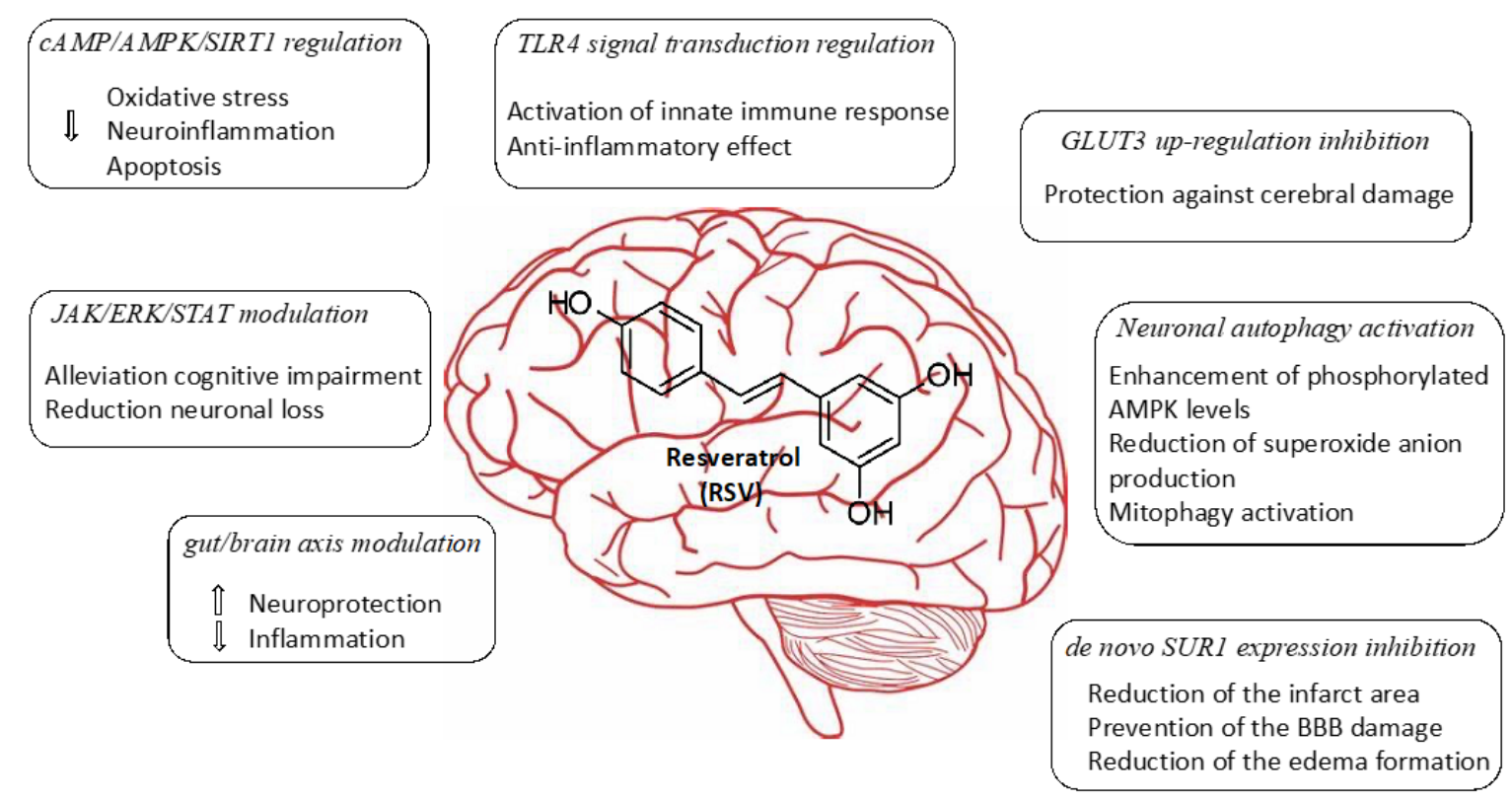

Figure 1. Potential mechanisms of action during an ischemic brain injury episode after treatment with RSV covered in this review.

\section{Neuroprotective Mechanism Associated with Treatments Involving Resveratrol \\ 2.1. Regulation of $c A M P / A M P K / S I R T 1$ Pathway}

Recently, dysfunction of energy metabolism has been considered as a possible important pathophysiological basis for cerebrovascular accidents. In this way, Wan et al. evaluated the neuroprotector action of RSV in cerebral ischemic injury [28]. Besides, the authors proposed a mechanism of action based on the regulation of energy metabolism, identifying potential targets of RSV. Results showed that treatment with RSV induced activation of AMPK and SIRT1 by inhibiting cyclic nucleotide PDEs.

For the development of the studies, in vivo cerebral ischemia injury was induced in 7-8-week-old male Sprague Dawley rats by middle cerebral artery occlusion (MCAO). After $2 \mathrm{~h}$, the cerebral obstruction was removed, and rats were reperfused. RSV dissolved in $5 \%$ dimethyl sulfoxide was repeatedly injected via intraperitoneal at $20 \mathrm{mg} / \mathrm{kg}$ once a day for 5 days before the occlusion procedure and for the final time before surgery. Results suggested a neuroprotective effect of RSV in MCAO rats since the degree of neurological deficits (based on a 5-point scale) significantly decreased from 2.75 for the control group to 1.67 after RSV treatment (Figure 2). Besides, these values further decreased with an increase in dose $(20 \mathrm{mg} / \mathrm{kg}$ to $30 \mathrm{mg} / \mathrm{kg})$. On the other hand, infarct volume measurements showed that RSV protected the rat brain since the volume measured was significantly lower than for the control group ( $31.5 \mathrm{~mm}^{3}$ vs. $43.8 \mathrm{~mm}^{3}$, respectively). Regarding the mechanism, the authors hypothesized that RSV might exert its neuroprotection by increasing SIRT1 and p-AMPK protein levels. In fact, results revealed that the protein expression of SIRT1 and p-AMPK were increased 1.8-fold and 1.4-fold, respectively, in comparison with the vehicle group. To establish the relationship between SIRT1 and p-AMPK in the signaling pathways mediated by RSV, authors employed dorsomorphin, an AMPK inhibitor, and sirtinol as SIRT1-specific inhibitors together with RSV. The first inhibitor produced a considerable decrease, not only in p-AMPK levels but also in SIRT1 levels, while the second inhibitor only affected SIRT1 levels without modifying AMPK levels. Based on that, the authors suggested that RSV could increase SIRT1 levels by activation of AMPK. 
A

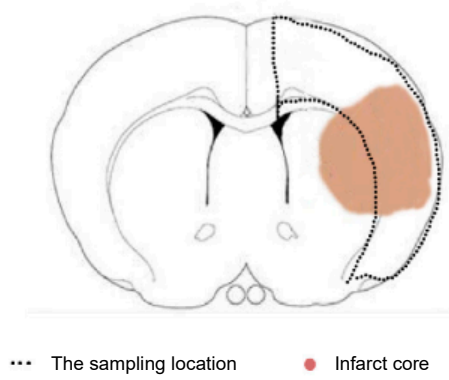

B

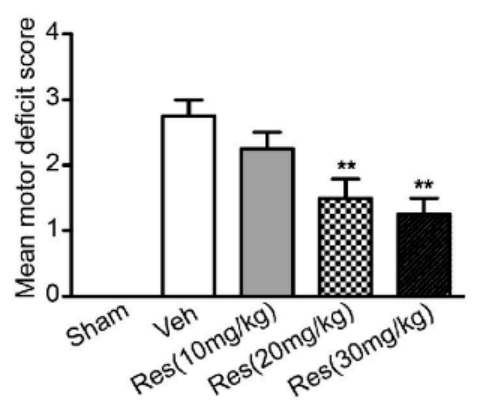

C
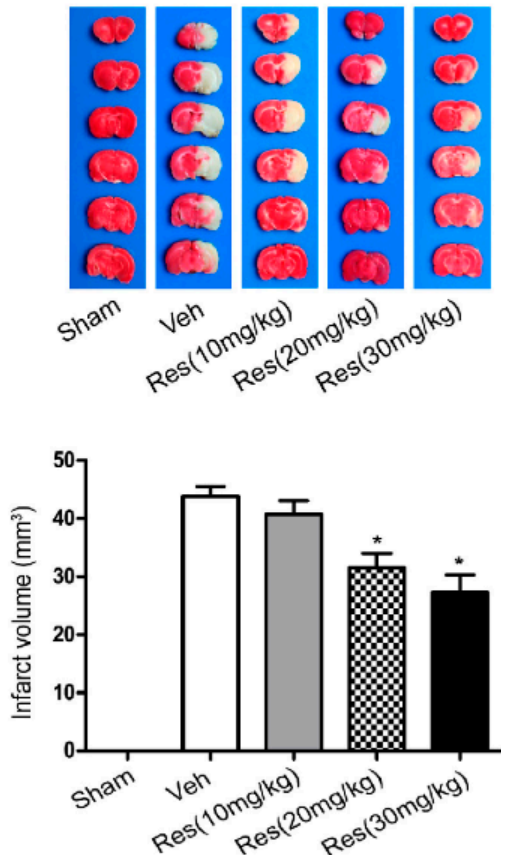

Figure 2. RSV provides neuroprotection $24 \mathrm{~h}$ after MCAO. (A) Schematic illustration of the sampling locations on the ipsilateral side of the cerebral cortex; (B) Neurological deficit scores following administration of $20 \mathrm{mg} / \mathrm{kg}$ and $30 \mathrm{mg} / \mathrm{kg}$ in the resveratrol treatment group were significantly decreased compared with the vehicle group; (C) 2,3,5-Triphenyltetrazolium chloride (TTC) staining of coronal sections and quantitative analysis of infarct regions $24 \mathrm{~h}$ after MCAO. Red and white represent normal and infarct tissue, respectively. Data are expressed as mean \pm S.E.M. $\left({ }^{*} p<0.05\right.$ and ** $p<0.01 ; n=4$ in each group). Adapted with permission from Elsevier (Wan, 2016).

The authors also used rolipram as a PDE inhibitor, and its neuroprotective activity was evaluated and compared with that of RSV. Results showed that both significantly increased ATP levels in MCAO rats compared with sham rats $(0.30 \mu \mathrm{mol} / \mathrm{g}$ vs. $0.21 \mu \mathrm{mol} / \mathrm{g})$. Additionally, rolipram increased the expression and levels of SIRT1 and AMPK proteins. Finally, RSV and rolipram inhibited PDE 4A expression, a representative PDE. Hence, the last results confirmed that RSV inhibited PDEs, specifically PDE4A, and as a result, it increases cAMP levels, and thus, the neuroprotective effect is promoted.

The beneficial effect of RSV in induced cerebral ischemia and reperfusion was demonstrated. A new insight into the molecular mechanism based on the regulation of neuronal metabolism was proposed. This is the first study that attributes RSV direct inhibition of PDEs and may provide a potential therapeutic target for ischemic stroke treatment. Nevertheless, further studies are needed to investigate the fundamental mechanism of SIRT1 and AMPK activation and the cAMP signaling.

\subsection{Modulating JAK/ERK/STAT Signaling Pathway}

To investigate the protective effect of RSV on hippocampus neurons from cerebral ischemia, Chang et al. administered RSV after an induced stroke. The authors proposed that RSV inhibited not only phosphorylation of Janus kinase (JAK)/extracellular signalregulated kinases (ERK)/signal traducers and activators of transcription (STAT), exerting a neuroprotective effect, but also promoted downregulation of inflammatory cytokines, reduced neuronal loss, and alleviated cognitive impairment [30].

The authors carried out in vivo studies inducing a cerebral ischemia-reperfusion injury in Sprague Dawley rats. The occlusion was maintained for $2 \mathrm{~h}$, and treatment started for 7 consecutive days using a concentration of $20 \mathrm{mg} / \mathrm{kg}$ of RSV dissolved in 50\% propylene glycol. The locomotive activity of rats was evaluated using open-field and closed- 
field tests after 7 days. One week later, the authors studied both memory and learning abilities using the Morris water maze (MWM) test. Then, 3 weeks later after injury, rats were sacrificed to conduct histology, Western blot assay, lipid peroxidation, and antioxidant enzyme assays. This study demonstrated that RSV was able to protect the neuronal loss in the hippocampus from ischemia/reperfusion injury via modulating the JAK/ERK/STAT signaling pathway. This was confirmed using hematoxylin-eosin (HE) staining by counting the number of necrotic neurons in the hippocampus. In addition, the authors showed that RSV prevented memory deficits in rats and effectively reduced lipid peroxidation involved in programmed cell death cascades and inflammation. Therefore, these preliminary results have served to underscore the potential of RSV in treating ischemia-reperfusion injury in the brain, but further experiments should be properly designed to launch preclinical studies to confirm this capability.

\subsection{Regulation of TLR4 Signal Transduction}

Neonatal hypoxic-ischemic encephalopathy (HIE), or hypoxic-ischemic brain injury (HIBI), is caused by disorders in cerebral blood flow and oxygen supply, being the most common cause of perinatal brain injury, which can lead to neonatal death or cause irreversible or prolonged physical and mental disabilities that lack appropriate treatment. Le et al. evaluated the activity of high mobility group box-1 (HMGB1) through the activation of SIRT1 by RSV in the inhibition of HI insult-induced neuroinflammation [31]. Using in vivo and in vitro assays, the authors confirmed that RSV inhibited the acetylation level and release of HMGB1, which was actively involved in the activation of the inflammatory cytokines signaling pathways (Toll-Like Receptor 4 (TLR4)/MyD88/Nuclear factor kappalight-chain-enhancer of activated B cells (NF- $\mathrm{kB})$ ), by increasing the expression and activity of SIRT1.

For this purpose, the in vivo experiments were performed using the Rice-Vannucci method. The hypoxic-ischemic brain injury was induced in postnatal day 7 pups, which were then placed in a hypoxia chamber for $1 \mathrm{~h}$. After the injury and $12 \mathrm{~h}$ later, RSV at a concentration of $100 \mathrm{mg} / \mathrm{mL}$ in PBS was administered via intraperitoneal injection using a volume of $45-55 \mu \mathrm{L} /$ mouse. Behavioral tests showed that the performance anomalies were significantly ameliorated after RSV administration. The Western blotting analysis from brain tissues and the conditioned medium of BV2 microglial cell culture indicated that RSV increased levels of SIRT1 and decreased TLR4, MyD88, and nuclear NF-kB. Besides, the authors observed changes in the localization of HMGB1 after injury. First, it was in the nucleus of the microglia, and then it was partially located out of the nucleus. Interestingly, RSV diminished the cytoplasmatic localization of HGMB1. Using mouse microglial BV2 cells after hypoxic-ischemic insult by oxygen-glucose deprivation (OGD), in vitro tests were performed to elucidate the role of TLR4 (the classical receptor of HMBG1). The stress produced by OGD significantly increased the mRNA expression and levels of cytokines (IL$1 \beta, \mathrm{IL}-6, \mathrm{TNF}-\alpha)$ and the expression of TLR4 and NF-KB. The in vitro results demonstrated that RSV also inhibited the amplification of inflammation at a concentration of $25 \mu \mathrm{M}$. The activation of SIRT1 due to RSV was confirmed using in vitro tests with stressed microglia cells and co-immunoprecipitation. The expression and deacetylase activity of SIRT1 as well as the interaction between SIRT1 and HGMB1 were increased by RSV (Figure 3). As a consequence, the level of acetylated HGMB1 and nucleocytoplasmatic translocation was inhibited. Finally, confocal imaging of BV2 cells evidenced the neuroprotective effect of RSV. So far, the specific mechanism for the treatment of neuroinflammation induced by hypoxicischemic insult in the immature brain was unclear. However, the authors described the action of essential factors such as HMGB1 and TLR4 involved in the neuroinflammation. Similarly, other authors also observed that RSV could inhibit the inflammation as a regulator of microglia/macrophages activation, reducing the production of inflammatory mediators in the ischemic cortex (IL-1 $\beta$, TNF- $\alpha$, and ROS). Thus, a neuroprotection effect was evidenced via reduction of glial cell activation and prevention of delayed neuronal cell death [24]. 


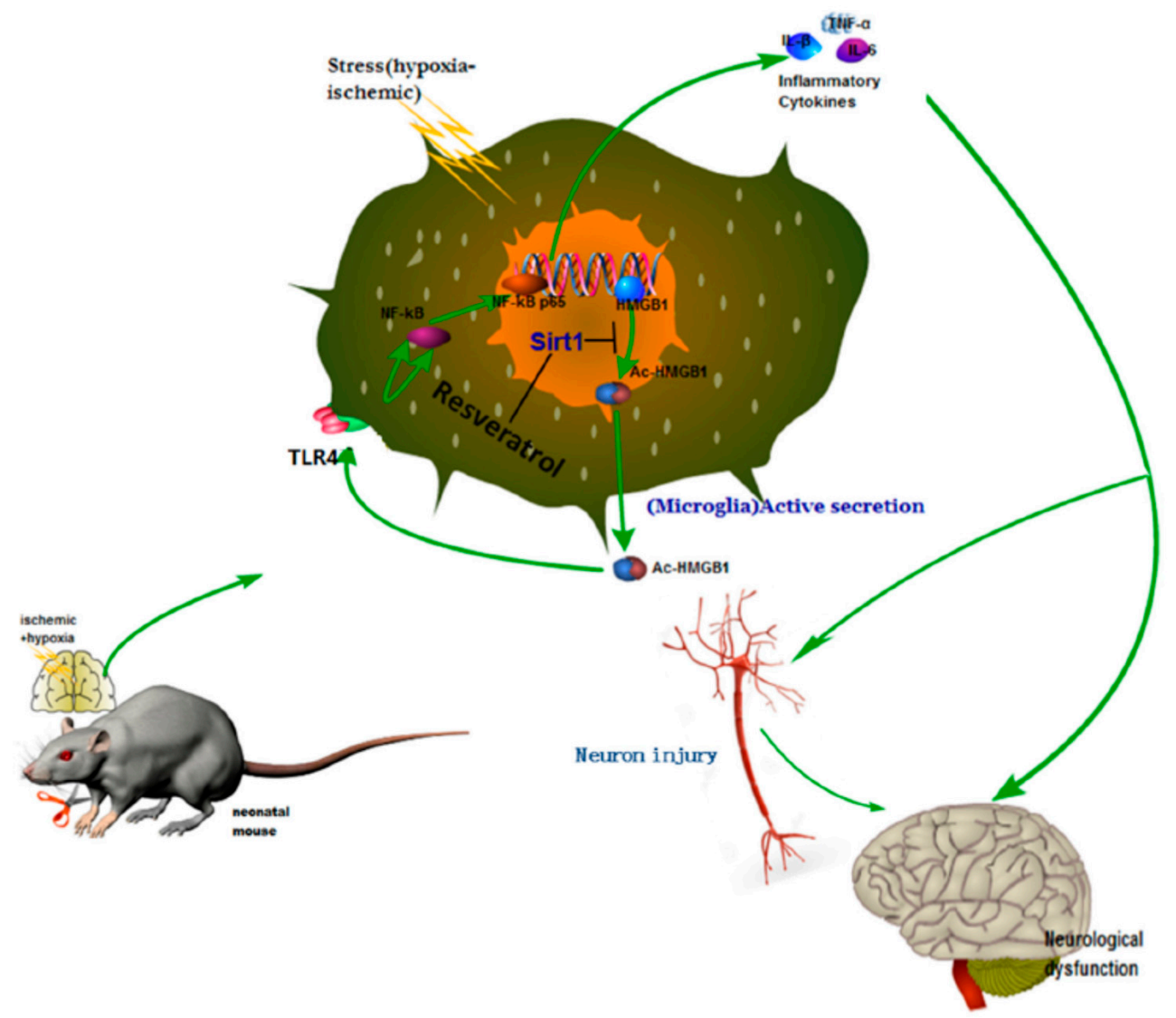

Figure 3. Schematic illustration of the anti-neuroinflammatory mechanism of RSV in HIBI involving the regulation of HMGB1 release by activated microglial SIRT1. As illustrated, HI insult-induced reactive microglia actively release acetylated HMGB1 (Ac-HMGB1), which in turn activates the TLR4/MyD88/NF-kB signaling pathway in microglia to initiate a glial-neuronal neuroinflammatory response by producing a wide array of pro-inflammatory factors and mediators such as IL-1 $\beta$, IL-6, and TNF- $\alpha$. RSV inhibits the acetylation of HMGB1 by activating SIRT1, thereby preventing the activation of the TLR4 signaling pathway by extracellular HMGB1. Reprinted with permission from Elsevier (Le, 2019).

In this same direction, other reports have reaffirmed the potent anti-inflammatory properties of RSV and also suggest its effect by modulating TLR4 expression in the HMGB1TLR4 signaling pathway [26]. Overall, these works represent a start point for the potential treatment of this high-incidence disorder based on the administration of RSV.

\subsection{Mechanism Targeting Gut/Brain Axis}

Inflammation and immune system activation are two important processes that can affect the brain after acute stroke. Importantly, emerging evidence has proved the role of the microbiota-gut brain axis in the ischemic brain. In this sense, Dou et al. demonstrated for the first time that RSV might inhibit systemic post-stroke inflammations, inducing neuroprotection by modulating intestinal-immune-cell-mediated inflammation [32]. The authors proposed one of the possible multifactorial mechanisms through which RSV could act is by modulating T helper 17 (Th17)/regulatory T (Treg) and Th1/Th2 cells as well as the expression of their related cytokines in the small intestine. 
The authors carried out in vivo studies by inducing focal cerebral ischemia in mice. This was produced by applying a transient occlusion in the middle cerebral artery (MCAO) for $60 \mathrm{~min}$. Then, extensive in vivo experiments were performed by dividing mice into nine experimental groups, including controls. Some groups were pre-treated with vancomycin, clavulanic acid, or amoxicillin during 3 days of previous MCAO. Additionally, fecal microbiota transplantation and RSV were administered intraperitoneally to experimental groups in order to confirm the neuroprotective effect of RSV, which was directly mediated by the intestinal flora. The RSV concentration used in this study was $200 \mathrm{mg} / \mathrm{kg}$. After 3 days of treatment, mice were sacrificed, and a series of quantification tests (e.g., flow cytometry, Western blotting, albumin quantification by ELISA, vascular permeability assay, and RNA extraction) were performed to measure the mRNA levels of two cytokines (IFN- $\gamma$ and IL-4) and Th17/Treg response. Interestingly, the authors found an important role of the gut-brain axis in acute ischemic stroke, demonstrating the neuroprotective effect of RSV when administered. Therefore, the authors showed that RSV was able to modulate Th1/Th2 balance and reduce small intestinal pro-inflammatory cytokines, including intestinal vascular permeability. The results also confirmed the ability of RSV to protect against systemic post-stroke inflammation in mice in the cortex and striatum.

\subsection{Inhibition of GLUT3 Up-Regulation}

After cerebral ischemia, the brain metabolic energy is reduced. In consequence, an up-regulation of glucose transporter (GLUT3) is an adaptive response for the rapid nutrient supply. Nonetheless, the fluctuation in glucose levels alters mitochondrial functionality, promotes apoptosis, and stimulates pro-inflammatory factors. On this basis, Gutiérrez Aguilar et al. studied the effect of RSV on GLUT3 expression levels after induced ischemia [33]. Results showed that the polyphenol protects neurons from middle cerebral artery occlusion damage and prevents GLUT3 up-regulation in the injured brain that could depend on AMPK activation.

In this study, male Wistar rats were subjected to MCAO for $2 \mathrm{~h}$, followed by different times of reperfusion $(1-24 \mathrm{~h})$. RSV $(1.9 \mathrm{mg} / \mathrm{kg})$ was administrated intravenously, diluted in ethanol at the onset of reperfusion. The infarct area measurements demonstrated that RSV reduced MCAO-induced damage since the expression levels of Microtubuleassociated protein 2 (MAP2), an indicator of surviving neurons, were conserved after the injury, ensuring the integrity of the fibers. Furthermore, RSV prevented the increment in GLUT3 mRNA stimulated by $2 \mathrm{~h}$ of MCAO and $1 \mathrm{~h}$ of reperfusion, while those levels greatly increased in the group without treatment (2.5-fold) (Figure 4). Besides, the same result was obtained for GLUT3 protein levels for $24 \mathrm{~h}$ of reperfusion and confirmed by immunofluorescence microscopy. The authors also evaluated the role of RSV in the inactivation of GLUT3 via p-AMPK, specifically through p65-NF- $\mathrm{kB}$, a regulator of GLUT3 expression. For this purpose, glutamate-induced excitotoxicity (model of ischemic stroke) was induced in mixed cultures of neurons and astrocytes. The authors found that RSV increased the phosphorylation of AMPK in both types of cells, indicating that this molecule not only induced an endogenous response but also activated a compensatory mechanism in astrocytes. Based on these results, the authors proposed a mechanism based on RSVtriggered phosphorylation of AMPK. This factor activates SIRT, which subsequently inhibits the transcriptional activity of p65-NF- $\mathrm{kB}$, which is involved in the GLUT3 regulation. However, this is still considered a preliminary study, and further research is essential to improve the comprehension of the process. 
A
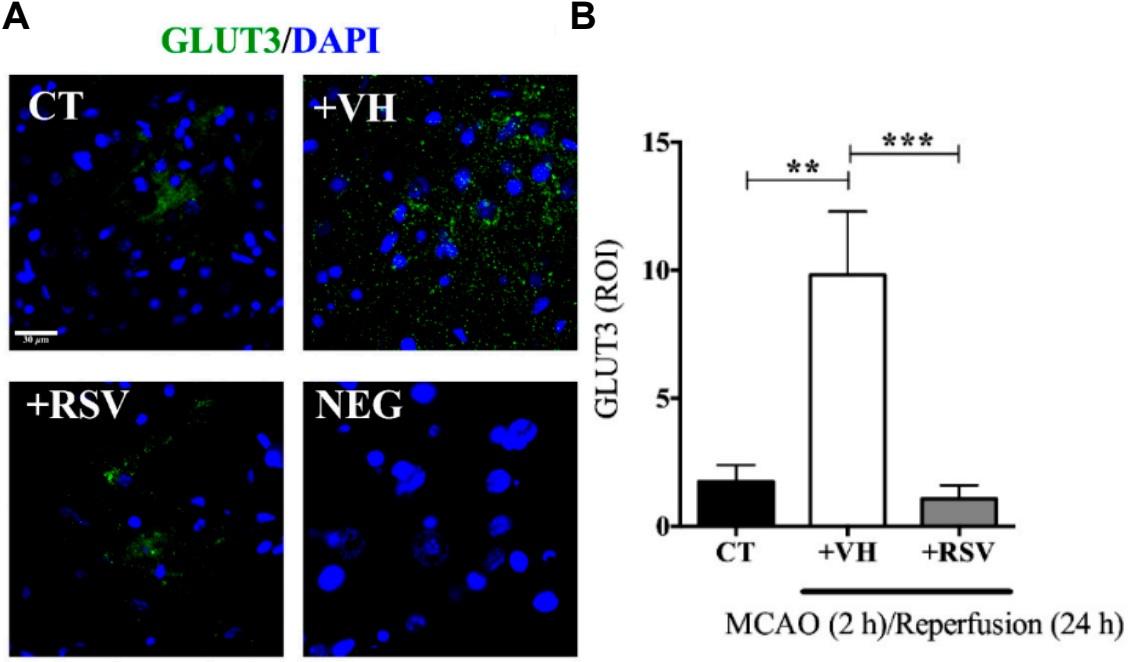

Figure 4. RSV prevents GLUT3 up-regulation induced in the brain after ischemia. Rats were subjected to MCAO for $2 \mathrm{~h}$ followed by restoration of blood flow (reperfusion) for $24 \mathrm{~h}$. Control animals (CT) were subjected to simulated MCAO. At the onset of restoration of blood flow, animals received either vehicle, ethanol $50 \%(+\mathrm{VH})$, or resveratrol at $1.9 \mathrm{mg} / \mathrm{kg}$, body weight $(+\mathrm{RSV})$. (A) Immunofluorescence of GLUT3 (green) protein expression. 4',6-diamidino-2-phenylindole (DAPI) was used to detect nuclei (blue); (B) Quantification of GLUT3 expression. Fluorescence was reported in \% of the signal associated with GLUT3 in the region of interest (ROI). Values of ROI were expressed as mean, \pm standard deviation. ANOVA, Tukey, ${ }^{* *} p<0.01,{ }^{* * *} p<0.001$. Adapted with permission from MDPI (Gutiérrez Aguilar, 2020).

\subsection{Activation of Neuronal Autophagy}

Based on the fact that AMPK preserves the cellular energy levels and may contribute to neuroprotection by activating autophagy after cellular stress such as cerebral ischemia or excitotoxicity, Pineda-Ramírez et al. investigated the neuroprotector effect of resveratrol on this via [34]. They demonstrated that RSV enhanced AMPK activity and drove autophagy, suggesting an increase of survival neurons. Herein, cerebral ischemia was induced in Wistar rats through the $\mathrm{MCAO}$ for $2 \mathrm{~h}$, and then reperfusion was induced. A single dose of RSV $(1.8 \mathrm{mg} / \mathrm{kg}$ in water-ethanol $50 \% \mathrm{v} / \mathrm{v})$ was administrated in the tail vein at the onset of reperfusion. Results showed that p-AMPK levels were significantly increased when RSV was administrated in comparison with the non-treated group. Besides, the infarct area detected was markedly reduced after treatment with RSV than for the control group $(20.76 \pm 1.67 \%$ and $28.08 \pm 2.84 \%$, respectively). The employment of an AMPK inhibitor, compound C ((6-[4-(2-piperidin-1-yl-ethoxy)-phenyl)]-3-pyridin-4-ylpyrrazolo [1,5-a]-pyrimidine), showed interference with the protective effect of RSV. For instance, the infarct area was reduced to only $25.7 \pm 2.25 \%$; the rat survival after $24 \mathrm{~h}$ of reperfusion was $57 \%$ and $90.47 \%$ for rats treated with RSV. This suggested that the neuroprotector effect of the polyphenol was partially related to AMPK activation.

Furthermore, the effect of RSV in autophagy through activation of AMPK was analyzed by the protein's expression levels involved in different stages of autophagy in neuronal cultures subjected to excitotoxicity. In relation to the first stages of autophagy, RSV increased Beclin 1 protein level, and thus, the microtubule-associated protein light chain 3 (LC3)-II/LC3-I ratio was significantly increased. Those proteins are involved in the formation of autophagosomes. In the final autophagy stage, p62 protein and lysosomalassociated membrane protein 1 (LAMP1) are important. In this case, RSV considerably reduced the level of LAMP1 without affecting p62 levels. The administration of compound $\mathrm{C}$ to prevent the AMPK activation from RSV produced the opposite effect. Thus, the authors demonstrated that RSV induced autophagy through AMPK activation. Specifically, mitochondrial degradation was studied through mitochondrial matrix protein HSP60 
expression. The expression of this protein was significant decreased after RSV treatment, and this effect was partially reversed by compound C. Based on this result, the authors proposed that the neuroprotector effect of RSV is due to the activation of AMPK, which leads to degradation of mitochondria by autophagy.

Looking for effective therapeutic agents for ischemic stroke treatment, Liu and coworkers studied the effect of combined therapy using RSV and rosuvastatin [35]. The combined pretreatment synergistically improved induced ischemic stroke. Besides, the authors proposed a possible in vivo mechanism based on the enhancement of anti-apoptosis, anti-inflammation, and autophagy. In the study, MCAO was induced in adult male Sprague Dawley rats for $90 \mathrm{~min}$. Then, the rats were reperfused. The administrations of drugs were carried out via intraperitoneal for RSV at a dose of $50 \mathrm{mg} / \mathrm{kg}$ and through intragastric gavage for rosuvastatin at a dose of $10 \mathrm{mg} / \mathrm{kg}$. The doses were given once a day for 7 repeated days before ischemia surgery. Interestingly, the pretreatment with a combination of drugs resulted in a stronger neuroprotective effect evidenced by cerebral infarct measurements and modified neurological severity scores (mNSS) after $24 \mathrm{~h}$ of arterial insult. The cerebral infarct volume significantly decreased in comparison with the control group from 0.3 to 0.1 , and those values were smaller than for separate therapy (for example, 0.2 for RSV only). Besides, the neurological score decreased considerably from 7 for the control group to 5 due to the combination of drugs. This result showed an improvement in motor, sensory, reflex, and balance of rats and, thus, less neurological damage. In relation to the mechanism, the anti-apoptosis activity was studied, measuring the apoptotic-associated proteins levels, such as Bax, Bcl-2, and caspase-3. The combination of drugs significantly decreased the expression of Bax and caspase 3, both apoptotic markers, compared to the control group (Figure 5). In contrast, the expression of Bcl-2, an anti-apoptotic factor, increased considerably. Thus, a more enhanced anti-apoptosis was observed by the synergic effect. The inflammatory response was also affected using the combined therapy. In fact, the expression of IL-1 $\beta$, an inflammatory factor, was remarkably decreased when compared to the separate pretreatment. Thus far, the strong neuroprotective effect was not only related to enhanced anti-apoptosis but also with a greater anti-inflammation. Lastly, the level of autophagy caused by ischemic brain injury was investigated, measuring the levels of LC3II, LC3I, and Beclin-1, all autophagy markers. The combination of drugs produced a considerable increase in Beclin-1 expression and the ratio of LC3-II/LC3-I in contrast with the drugs alone. Thus, neuroprotection was also associated with the upregulation of autophagy levels. Finally, a synergistic effect was clearly observed by the co-administration of a statin and the phytoalexin RSV. This neuroprotective effect was associated with a complex activation that included anti-apoptosis, anti-inflammation, and autophagy.

Other authors also suggested that RSV could induce autophagy as a mechanism of neuroprotection. He et al. hypothesized that RSV treatment could relieve cerebral ischemic injury by inhibiting NLRP3 inflammasome activation through induction of SIRT1dependent autophagy [36]. The NLRP3 is a NOD-like receptor belonging to the family of pyrin domain-containing 3 inflammasome which is involved in innate immunity and inflammation. After their stimulation, inflammasomes are activated and lead to caspase-1 activation with the release of bioactive cytokines (IL-1 $\beta$ and IL-18). The induction of autophagy could reduce the inflammasome-derived inflammation and exert the neuroprotective effect. The authors employed healthy male Sprague Dawley rats for the MCAO procedure during $1 \mathrm{~h}$ followed by $24 \mathrm{~h}$ reperfusion. RSV was injected at a dose of $100 \mathrm{mg} / \mathrm{kg}$ (i.p.) at the onset of reperfusion. The results suggested that RSV can ameliorate cerebral ischemic injury in rats since the infarct volume of the RSV-treated group was significantly decreased, the neurological scores were remarkably improved, and the brain water content after reperfusion was reduced in comparison with the non-treated group. Moreover, the levels of NLRP3 inflammasome-regulated cytokines such as caspase-1, IL-1 $\beta$, and IL-18 were significant inhibited by treatment with RSV. In addition, the protein levels of SIRT1 were upregulated when RSV was administrated in comparison with the MCAO group. On the other hand, the autophagy markers such as LC3B-II/LC3B-I ratio and expression of p62 
were increased in the presence of the ischemic injury. The treatment with RSV increased the levels of LC3B-II/LC3B-I ratio even more and decreased the expression of p62, indicating an enhancement of autophagy activity. Finally, to assess if autophagy activity and NLRP3 inflammasome activation were mediated by SIRT1, the SIRT1 siRNA pretreatment (along with or without RSV) was employed. The results showed a significant reverse of RSV effects such as a decrease in SIRT1 expression and in the LC3B-II/LC3B-I ratio and an increase in the expression of p62, i.e., inhibition of autophagy. Besides, the downregulation of NLRP3 inflammasomes by RSV was markedly inhibited by the SIRT1 knockdown. In this way, the authors confirmed that the effect of RSV on autophagy and the inhibition of NLRP3 inflammasome was regulated by the SIRT1 pathway. Thus, these findings demonstrated that RSV administration results are beneficial as a treatment of cerebral ischemic in rats; however, more details of the pathological mechanisms of this injury are required.

A

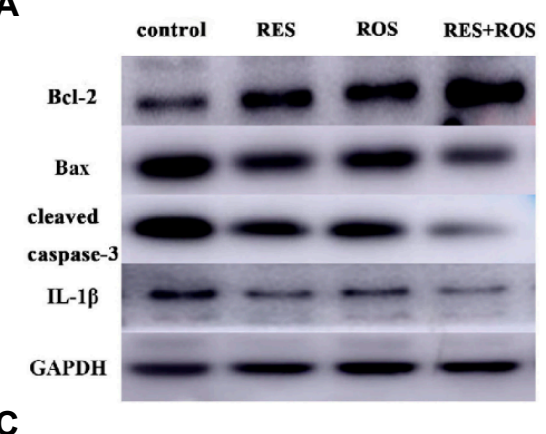

C

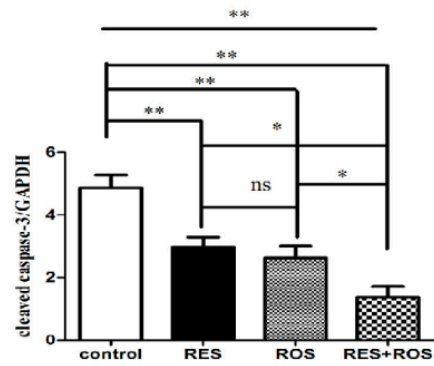

B

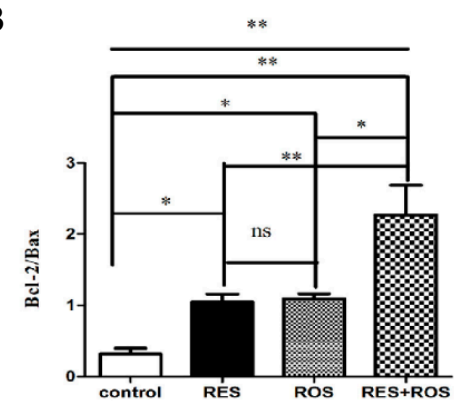

D

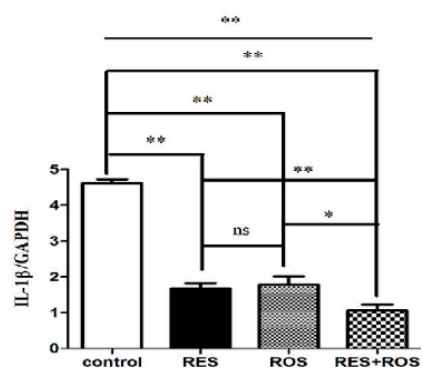

Figure 5. Synergistic effects of combined resveratrol (note: in this figure, it is abbreviated as RES) and rosuvastatin (note: in this figure, it is abbreviated as ROS) pretreatment on cell apoptosis and inflammatory response caused by cerebral I/R injury. (A) Representative protein expression of Bcl-2, Bax, caspase-3, and IL-1 $\beta$, examined by Western blot at $24 \mathrm{~h}$ after ischemia onset; (B-D) The bar graphs show that RES alone, ROS alone, and combined RES and ROS pretreatment significantly induced the ratio of Bcl-2/Bax (B) and reduced the expressions of caspase-3 (C) and IL-1 $\beta$ (D) compared to the control group. More importantly, the bar graphs show that the ratio of $\mathrm{Bcl}-2 / \mathrm{Bax}(\mathbf{B})$ of group receiving combined RES and ROS pretreatment was significantly increased compared with the group receiving RES alone or ROS alone pretreatment, whereas the expressions of caspase-3 (C) and IL-1 $\beta$ (D) of group receiving combined RES and ROS pretreatment were significantly decreased compared to the group receiving RES alone or ROS alone pretreatment. In addition, the bar graphs also show that there were no significant (ns) differences in the ratio of $\mathrm{Bcl}-2 / \mathrm{Bax}(\mathbf{B})$ and the expressions of caspase-3 (C) and IL-1 $\beta$ (D) between group receiving RES pretreatment and group receiving ROS pretreatment. One-factor analysis of variance was used to assess statistical significance between means $\left({ }^{* *} p<0.01 ;{ }^{*} p<0.05\right)$. ns: not significant. Adapted with permission from Elsevier (Liu, 2018).

\subsection{Via De Novo SUR1 Expression Inhibition}

Another potential mechanism in which RSV has shown effectiveness is in reducing the infarct area and cerebral edema, resulting in an improvement of the neurological performers, reduction of the blood-brain barrier (BBB) damage, and increase of the survival acting in the process of inhibiting Abcc 8 gene transcription that codes for Sulfonylurea receptor 1 (SUR1). 
This study published by Alquisiras-Burgos et al. assessed in vivo studies using male Wistar rats that were divided into three groups: control (CT), vehicle (VH) ischemic group, and the treatment group with RSV [37]. Then, the authors proceeded to occlude the middle cerebral artery for $2 \mathrm{~h}$. At the beginning of reperfusion, RSV was injected $(1.9 \mathrm{mg} / \mathrm{kg})$ in the tail vein. Before rats were sacrificed, the "limb-use asymmetry test" was performed to evaluate the neurological performance of the animals at $24 \mathrm{~h}$ of reperfusion. Then, the permeability of $\mathrm{BBB}$ was evaluated, and the individual hemisphere water change content (\%) was quantified by adding Evans blue ( $2 \%$ ) diluted in saline during reperfusion $(4 \mathrm{~mL} / \mathrm{kg})$ and observing the color change in the tissue. Likewise, the authors quantified the infarct area by TTC staining assay, which showed the highest effect in the somatosensory cortex. Interestingly, the binding activity of specificity protein 1 (SP1) transcription factor was evaluated using electrophoretic mobility shift assays (EMSA) with $5 \mu \mathrm{g}$ of nuclear extract and $0.2 \mu \mathrm{g}$ of biotin-labeled oligonucleotides mixed with binding reagents. In addition, immunofluorescence assays with a specific endothelial marker and Western blot assays to quantify SUR1 and Aquaporin-4 (AQP4) expression were assessed (Figure 6). Despite the fact that a precise mechanism is still unclear, the results in animal models showed that RSV had a tendency to target SP transcription factors and inhibit the expression of SUR1 and AQP4. As a result, the authors found that RSV significantly protected BBB integrity and increased the rate of survival from $61.9 \%$ to $90.4 \%$, attenuating infarct volumes. Alternatively, the authors found evidence that the effect of RSV on regulating the balance between matrix metalloproteinase (MMP)-9 and the inhibitor TIMP-1 might prevent the BBB disruption after ischemic stroke.

A

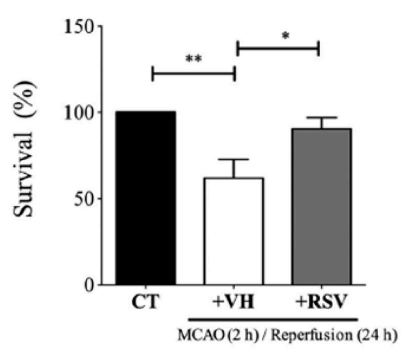

C

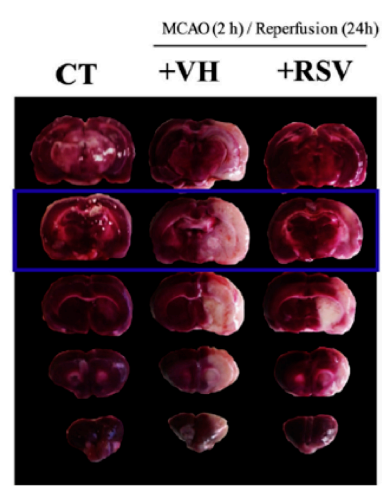

B

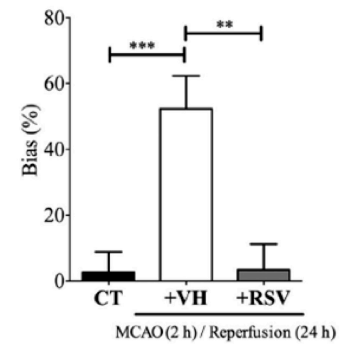

D

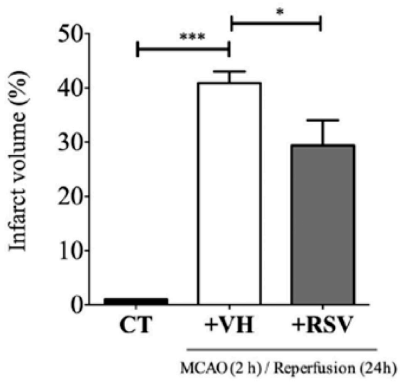

Figure 6. Animals were subjected to $2 \mathrm{~h}$ of MCAO followed by $24 \mathrm{~h}$ of reperfusion. CT animals underwent simulated MCAO. At the onset of reperfusion animals received either vehicle $(+\mathrm{VH}$, ethanol 50\%; i.v.) or RSV (+RSV, $1.9 \mathrm{mg} / \mathrm{kg}$, body weight; i.v.). (A) Mortality assessed during the $24 \mathrm{~h}$ followed MCAO; (B) Neurological status of the animals calculated by the Bias "limb-use asymmetry test"; (C) Infarcted area assessed using TTC staining method in coronal slices of brains; (D) Infarct area quantified on the slice located approximately at Bregma -2.16 to $-4.20 \mathrm{~mm}$. Values were expressed as mean \pm SD. ANOVA, Tukey, ${ }^{*} p<0.05,{ }^{* *} p<0.01,{ }^{* *} p<0.001$. Adapted with permission from Elsevier (Alquisiras-Burgos, I. 2020). 


\subsection{Administration of Resveratrol-Loaded Nanoparticles (NPs)}

In addition to using RSV itself as a therapeutic molecule to treat ischemic brain injuries, other strategies have been assessed. Lu et al. prepared NPs made up of poly $(\mathrm{N}-$ vinylpyrrolidone)- $b$-poly( $\varepsilon$-caprolactone) (PVP-PCL) as drug carriers for RSV [38]. The resulting particles exhibited spherical shape and diameters of $100 \mathrm{~nm}$, according to Scanning Electron Microscopy (SEM) and Dynamic Light Scattering (DLS), respectively. In vitro controlled release experiments were performed. These assays showed an initial burst release of $37 \%$ and sustained release of RSV close to $65 \%$ after 6 days.

The in vivo effect in model rats was confirmed with different experimental groups. In addition to control groups (saline, empty NPs, and $10 \mathrm{mg} / \mathrm{kg}$ unloaded RSV), increasing concentrations of RSV-loaded NPs $(1,5$ and $10 \mathrm{mg} / \mathrm{kg})$ were tested. The administration of RSV-loaded NPs was carried out via carotid artery rather than tail vein as this approach showed an increase of the circulating NPs in the cerebral vasculature. Once the parameters were optimized in terms of drug dose $(5 \mathrm{mg} / \mathrm{kg})$, the authors investigated the leakage of the BBB with Evan blue $(2.5 \mathrm{~mL} / \mathrm{kg})$ dissolved in saline $(4 \% \mathrm{w} / \mathrm{v})$. TTC staining assay was also assessed and confirmed the infarct area was reduced by $31.7 \%$ and $34.1 \%$ after $24 \mathrm{~h}$ and $72 \mathrm{~h}$ of reperfusion, respectively. In addition, the level of lipid peroxidation was reduced by $56.5 \%$, approximately confirming the reduction of neuronal apoptosis. Finally, this study demonstrated that NPs were able to facilitate the release of RSV in vivo as well as a significant protection against cerebral ischemia/reperfusion, oxidative stress attenuation, and the prevention of brain edema.

\section{Conclusions}

This review has collected various recent studies describing the evaluation of the RSV effectiveness in the treatment of ischemic brain injury in different rodent animal models. RSV itself shares common properties with other polyphenol derivatives such as neuroprotection, anti-apoptotic, anti-inflammatory, and antioxidant characteristics. This has allowed RSV to be considered a promising small molecule drug for the treatment of agerelated disorders. In this regard, data experiments collected from recent studies have shown different mechanisms of action in which RSV plays important roles in the management of the disease, such as CAMP/AMPK/SIRT1 regulation, JAK/ERK/STAT signaling pathway modulation, TLR4 signal transduction regulation gut/brain axis modulation, GLUT3 up-regulation inhibition, neuronal autophagy activation, and de novo SUR1 expression inhibition. The in vivo experiments that have been selected for this review may open novel perspectives and challenges for ischemic brain injuries with the potential to be translated from bench to bedside. While RSV has exhibited remarkable activities when it has been systemically administered, its poor bioavailability makes this drug unsuitable for clinical use. Interestingly, novel encapsulation strategies and the use of nanotechnology have emerged to overcome these drawbacks and might facilitate the transport of RSV at a specific site of action. Despite the fact that there are still no effective drugs approved for the treatment of cerebral ischemia, additional and detailed preclinical experiments should be performed involving a range of animal models with the aim to afford the most efficient therapy strategies, including new encapsulation techniques and the use of RSV-load NPs.

Author Contributions: Conceptualization, D.D.D. and M.A.F.; methodology, N.D.M., G.V.A., M.A.F. and D.D.D., writing — original draft preparation, N.D.M., G.V.A., M.A.F., S.G. and D.D.D.; writingreview and editing, N.D.M., G.V.A., M.A.F., S.G. and D.D.D.; funding acquisition, D.D.D. and M.A.F. All authors have read and agreed to the published version of the manuscript.

Funding: This research was funded by the University of Regensburg, the Spanish Government (PID2019-105391GB-C21), Consejo Nacional de Investigaciones Científicas y Técnicas (CONICET, PIP 112-2015-01-00242), and Universidad Nacional de Córdoba (SECyT-UNC CONSOLIDAR 20182021, Código: 33620180100367CB).

Institutional Review Board Statement: Not applicable. 
Informed Consent Statement: Not applicable.

Acknowledgments: N.D.M. thanks Consejo Nacional de Investigaciones Científicas y Técnicas (CONICET) for the fellowship granted. MAF holds a researcher position at CONICET. D.D.D. thanks the Deutsche Forschungsgemeinschaft (DFG) for a Heisenberg Professorship Award and the Spanish Ministry of Science, Innovation and Universities for the Senior Beatriz Galindo Award (Distinguished Researcher). D.D.D. also thanks NANOtec, INTech, Cabildo de Tenerife, and ULL for laboratory facilities.

Conflicts of Interest: The authors declare no conflict of interest.

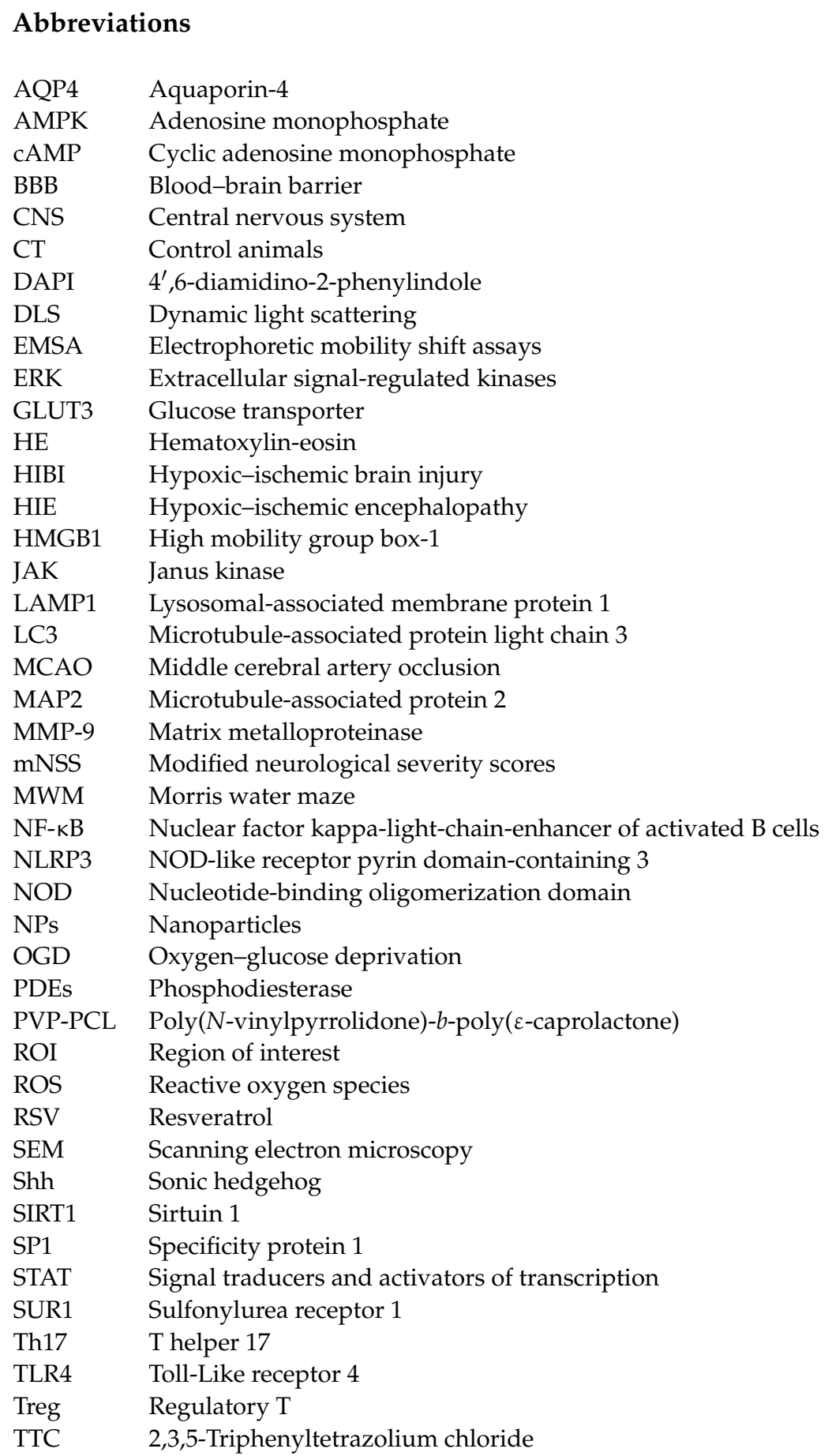




\section{References}

1. Summerlin, N.; Soo, E.; Thakur, S.; Qu, Z.; Jambhrunkar, S.; Popat, A. Resveratrol nanoformulations: Challenges and opportunities. Int. J. Pharm. 2015, 479, 282-290. [CrossRef]

2. Diaz-Gerevini, G.T.; Repossi, G.; Dain, A.; Tarres, M.C.; Das, U.N.; Eynard, A.R. Beneficial action of resveratrol: How and why? Nutrition 2016, 32, 174-178. [CrossRef]

3. Singh, A.P.; Singh, R.; Verma, S.S.; Rai, V.; Kaschula, C.H.; Maiti, P.; Gupta, S.C. Health benefits of resveratrol: Evidence from clinical studies. Med. Res. Rev. 2019, 39, 1851-1891. [CrossRef]

4. Raj, P.; Lieben Louis, X.; Thandapilly, S.J.; Movahed, A.; Zieroth, S.; Netticadan, T. Potential of resveratrol in the treatment of heart failure. Life Sci. 2014, 95, 63-71. [CrossRef]

5. Elshaer, M.; Chen, Y.; Wang, X.J.; Tang, X. Resveratrol: An overview of its anti-cancer mechanisms. Life Sci. 2018, 207, 340-349. [CrossRef]

6. Delmas, D.; Cornebise, C.; Courtaut, F.; Xiao, J.; Aires, V. New highlights of resveratrol: A review of properties against ocular diseases. Int. J. Mol. Sci. 2021, 22, 1295. [CrossRef]

7. Kulkarni, S.S.; Cantó, C. The molecular targets of resveratrol. Biochim. Biophys. Acta 2015, 1852, 1114-1123. [CrossRef]

8. Rauf, A.; Imran, M.; Suleria, H.A.R.; Ahmad, B.; Petersf, D.G.; Mubarak, M.S. A comprehensive review of the health perspectives of resveratrol. Food Funct. 2017, 8, 4284-4305. [CrossRef]

9. Huang, J.; Huang, N.; Xu, S.; Luo, Y.; Li, Y.; Jin, H.; Yu, C.; Shi, J.; Jin, F. Signaling mechanisms underlying inhibition of neuroinflammation by resveratrol in neurodegenerative diseases. J. Nutr. Biochem. 2021, 88, 108552. [CrossRef]

10. Ding, Z.; Cao, J.; Shen, Y.; Zou, Y.; Yang, X.; Zhou, W.; Guo, Q.; Huang, C. Resveratrol promotes nerve regeneration via activation of p300 acetyltransferase-mediated VEGF signaling in a rat model of sciatic nerve crush injury. Front. Neurosci. 2018, $12,341$. [CrossRef]

11. Zhang, S.; Botchway, B.O.A.; Zhang, Y.; Liu, X. Resveratrol can inhibit Notch signaling pathway to improve spinal cord injury. Ann. Anat. 2019, 223, 100-107. [CrossRef] [PubMed]

12. Tang, K.S.; Tan, J.S. The protective mechanisms of polydatin in cerebral ischemia. Eur. J. Pharmacol. 2019, 842, 133-138. [CrossRef]

13. Tressera-Rimbau, A.; Arranz, S.; Eder, M.; Vallverdú-Queralt, A. Dietary polyphenols in the prevention of stroke. Oxid. Med. Cell. Longev. 2017, 2017, 7467962. [CrossRef]

14. Zou, L.; Gong, Y.; Liu, S.; Liang, S. Natural compounds acting at P2 receptors alleviate peripheral neuropathy. Brain Res. Bull. 2019, 151, 125-131. [CrossRef]

15. Cheng, Y.C.; Sheen, J.M.; Hu, W.L.; Hung, J.C. Polyphenols and Oxidative Stress in Atherosclerosis-Related Ischemic Heart Disease and Stroke. Oxid. Med. Cell. Longev. 2017, 2017, 8526438. [CrossRef]

16. Yao, Y.; Zhang, Y.; Liao, X.; Yang, R.; Lei, Y.; Luo, J. Potential therapies for cerebral edema after ischemic stroke: A mini review. Front. Aging Neurosci. 2021, 12, 618819. [CrossRef]

17. Shao, A.; Lin, D.; Wang, L.; Tu, S.; Lenahan, C.; Zhang, J. Oxidative stress at the crossroads of aging, stroke and depression. Aging Dis. 2020, 11, 1537-1566. [CrossRef]

18. Long, Y.; Yang, Q.; Xiang, Y.; Zhang, Y.; Wan, J.; Liu, S.; Li, N.; Peng, W. Nose to brain drug delivery-A promising strategy for active components from herbal medicine for treating cerebral ischemia reperfusion. Pharmacol. Res. 2020, 159, 104795. [CrossRef]

19. Parrella, E.; Gussago, C.; Porrini, V.; Benarese, M.; Pizzi, M. From preclinical stroke models to humans: Polyphenols in the prevention and treatment of stroke. Nutrients 2021, 13, 85. [CrossRef]

20. López, M.; Dempsey, R.J.; Vemuganti, R. Resveratrol neuroprotection in stroke and traumatic CNS injury. Neurochem. Int. 2015, 89, 75-82. [CrossRef] [PubMed]

21. Chen, S.; Chen, H.; Du, Q.; Shen, J. Targeting myeloperoxidase (MPO) mediated oxidative stress and inflammation for reducing brain ischemia injury: Potential application of natural compounds. Front Physiol. 2020, 11, 433. [CrossRef] [PubMed]

22. Mohsenpour, H.; Pesce, M.; Patruno, A.; Bahrami, A.; Pour, P.M.; Farzaei, M.H. A review of plant extracts and plant-derived natural compounds in the prevention/treatment of neonatal hypoxic-ischemic brain injury. Int. J. Mol. Sci 2021, 22, 833. [CrossRef]

23. Reyes-Corral, M.; Sola-Idígora, N.; de la Puerta, R.; Montaner, J.; Ybot-González, P. Nutraceuticals in the prevention of neonatal hypoxia-ischemia: A comprehensive review of their neuroprotective properties, mechanisms of action and future directions. Int. J. Mol. Sci. 2021, 22, 2524. [CrossRef]

24. Subedi, L.; Gaire, B.P. Phytochemicals as regulators of microglia/macrophages activation in cerebral ischemia. Pharmacol. Res. 2021, 165, 105419. [CrossRef]

25. Rahimifard, M.; Maqbool, F.; Moeini-Nodeh, S.; Niaz, K.; Abdollahi, M.; Braidy, N.; Nabavi, S.M.; Nabavi, S.F. Targeting the TLR4 signaling pathway by polyphenols: A novel therapeutic strategy for neuroinflammation. Ageing Res. Rev. 2017, 36, 11-19. [CrossRef] [PubMed]

26. Tresserra-Rimbau, A.; Lamuela-Raventos, R.; Moreno, J.J. Polyphenols, food and pharma. Current knowledge and directions for future research. Biochem. Pharmacol. 2018, 156, 186-195. [CrossRef]

27. Yu, P.; Wang, L.; Tang, F.; Zeng, L.; Zhou, L.; Song, X.; Jia, W.; Chen, J.; Yang, Q. Resveratrol pretreatment decreases ischemic injury and improves neurological function via Sonic Hedgehog signaling after stroke in rats. Mol. Neurobiol. 2017, 54, 212-226. [CrossRef] [PubMed]

28. Wan, D.; Zhou, Y.; Wang, K.; Hou, Y.; Hou, R.; Ye, X. Resveratrol provides neuroprotection by inhibiting phosphodiesterases and regulating the cAMP/AMPK/SIRT1 pathway after stroke in rats. Brain Res. Bull. 2016, 121, 255-262. [CrossRef] 
29. Andrade, S.; Ramalho, M.J.; Pereira, M.C.; Loureiro, J.A. Resveratrol brain delivery for neurological disorders, prevention and treatment. Front. Pharmacol. 2018, 9, 1261. [CrossRef]

30. Chang, C.; Zhao, Y.; Song, G.; She, K. Resveratrol protects hippocampal neurons against cerebral ischemia-reperfusion injury via modulating JAK/ERK/STAT signaling pathways in rats. J. Neuroimmunol. 2018, 315, 9-14. [CrossRef]

31. Le, K.; Daliv, E.C.; Wu, S.; Qian, F.; Ali, A.I.; Yu, D.; Guo, Y. SIRT1-regulated HMGB1 release is partially involved in TLR4 signal transduction: A possible anti-neuroinflammatory mechanism of resveratrol in neonatal hypoxic-ischemic brain injury. Int. Immunopharmacol. 2019, 75, 105779. [CrossRef] [PubMed]

32. Dou, Z.; Rong, X.; Zhao, E.; Zhang, L.; Lv, Y. Neuroprotection of resveratrol against focal cerebral ischemia/reperfusion injury in mice through a mechanism targeting gust-brain axis. Cell. Mol. Neurobiol. 2019, 39, 883-898. [CrossRef] [PubMed]

33. Gutiérrez Aguilar, G.F.; Alquisiras-Burgos, I.; Franco-Pérez, I.; Pineda-Ramírez, N.; Ortiz-Plata, A.; Torres, I.; Pedraza-Chaverri, J.; Aguilera, P. Resveratrol prevents GLUT3 up-regulation induced by middle cerebral artery occlusion. Brain Sci. 2020, 10, 651. [CrossRef]

34. Pineda-Ramírez, N.; Alquisiras-Burgos, I.; Ortiz-Plata, A.; Ruiz-Tachiquín, M.E.; Espinoza-Rojo, M.; Aguilera, P. Resveratrol activates neuronal autophagy through AMPK in the ischemic brain. Mol. Neurobiol. 2020, 57, 1055-1069. [CrossRef] [PubMed]

35. Liu, Y.; Yang, H.; Jia, G.; Li, L.; Chen, H.; Bi, J.; Wang, C. The synergistic neuroprotective effects of combined rosuvastatin and resveratrol pretreatment against cerebral ischemia/reperfusion injury. J. Stroke Cerebrovasc. Dis. 2018, 27, 1697-1704. [CrossRef]

36. He, Q.; Li, Z.; Wang, Y.; Hou, Y.; Li, L.; Zhao, J. Resveratrol alleviates cerebral ischemia/reperfusion injury in rats by inhibiting NLRP3 inflammasome activation through Sirt1-dependent autophagy induction. Int. Immunopharmacol. 2017, 50, 208-215. [CrossRef]

37. Alquisiras-Burgos, I.; Ortiz-Plata, A.; Franco-Pérez, J.; Millán, A.; Aguilera, P. Resveratrol reduces cerebral edema through inhibition of de novo SUR1 expression induced after focal ischemia. Exp. Neurol. 2020, 330, 113353. [CrossRef]

38. Lu, X.; Dong, J.; Zheng, D.; Li, X.; Ding, D.; Xu, H. Reperfusion combined with intraarterial administration of resveratrol-loaded nanoparticles improved cerebral ischemia-reperfusion injury in rats. Nanomed. Nanotech. Biol. Med. 2020, 28, 102208. [CrossRef] 